\title{
EL DESPOBLAMIENTO DE LA PUNA: EFECTOS DEL CAMBIO CLIMÁTICO Y OTROS FACTORES ${ }^{1}$
}

\author{
Eyner Alata \\ Beatriz Fuentealba \\ Jorge Recharte \\ Instituto de Montaña
}

\begin{abstract}
Resumen:
Establecer con precisión el contexto en el que se realizan acciones de adaptación del cambio climático es muy importante puesto que nos permite identificar factores más allá de los ecológicos. En un trabajo de campo realizado en dos comunidades de la Cordillera Blanca, Canray Grande y Los Andes de Recuay, identificamos un despoblamiento en la puna. En sectores donde antes (decádas de los 70 y 80) habitaban diez o más familias, ahora solo quedan tres familias. Para conocer las razones o causas de este despoblamiento usamos el trabajo de campo etnográfico que nos permitió ver que este despoblamiento obedece a factores que van más allá del cambio climático.
\end{abstract}

Identificamos que este despoblamiento se debe a características demográficas propias de estas familias de puna como su ciclo de vida, que está orientado al abandono de la puna. Las familias pastoras usan el ganado como un activo, inicialmente como una forma de acumulación y luego de consolidación. Sin embargo, a medida que envejecen, disminuyen el número de sus animales y abandonan la puna; mientras las nuevas generaciones perciben el pastoreo como una actividad que los mantiene pobres y es muy sacrificada, orientándose a actividades asalariadas fuera de la puna.

Palabras clave:

Etnografía, contextualización, pastores, campesinos, Ancash.

1 Ponencia presentada en el Foro Internacional de Ciencias Sociales Cambio Climático. Desastres y Gobernanza realizado en Cusco del 2 al 5 de agosto de 2017. 


\title{
Depopulation in the Puna: Effects of Climate Change and other Factors
}

\begin{abstract}
:
Precisely establishing the context in which adaptation to climate change is occurring is very important, as it allows us to identify factors beyond the ecological. In fieldwork conducted in two communities in the Cordillera Blanca, Canray Grande and Los Andes Recuay, we have identified population loss in the puna. In sectors where before (1970s/80s) there were ten or more families, there are now only three families. To understand the reasons or causes of this population loss, we used ethnographic fieldwork, which revealed that this decline is due to factors beyond climate change.
\end{abstract}

We attribute this population loss to demographic characteristics specific to these families in the puna, such as their lifecycles, which propagate the abandonment of the puna. Pastoral families use livestock as an asset, initially as a form of accumulation, and later as a form of consolidation. However, as families age, the number of animals they own declines and they abandon the puna. Concurrently, new generations view herding as a poverty trap, and as very demanding work, therefore orienting themselves towards wage-earning activities outside of the puna.

\section{Keywords:}

Ethnography, contextualization, patoralism, peasants, Ancash. 


\section{Eyner Alata}

Licenciado en Antropología por la Pontificia Universidad Católica del Perú, con intereses de investigación sobre temas de campesinado, minería artesanal, desarrollo rural y conservación. Actualmente es investigador asociado del Instituto de Montaña.

Correo: eyner.alata@pucp.pe

\section{Beatriz Fuentealba}

Doctora y Magister en Biología por la Universidad Nacional Autónoma de México y Licenciada en Biología por la Universidad Nacional Agraria de La Molina. Hace más de diez años viene investigando temas que vinculan la restauración ecológica y agroforestal integradas al manejo de ecosistemas. Actualmente es la coordinadora del área de Restauración de Ecosistemas de Alta Montaña del Instituto de Montaña.

Correo: bfuentealba@mountain.org

\section{Jorge Recharte}

Doctor y Magister en Antropología por la Universidad de Cornell y Licenciado en Antropología por la Pontificia Universidad Católica del Perú. Desde 1997 ha liderado investigaciones y programas de conservación de ecosistemas de montaña y afirmación cultural en los Andes del Perú y Ecuador con instituciones como el Instituto de Montaña y FLACSO, entre otros. Actualmente es director del Instituto de Montaña en el Perú.

Correo: jrecharte@mountain.org 


\section{Introducción}

Existen numerosas iniciativas públicas y privadas, normativas y de acción, que promueven en el Perú la adaptación al cambio climático. Este universo de iniciativas de adaptación dirigida sigue las pautas del Grupo Inter-Gubernamental de Expertos sobre Cambio Climático (IPCC, por sus siglas en inglés) y se refiere a dar respuestas relacionadas directamente con los impactos originados por cambios en los promedios históricos de las variables climáticas, sean actuales o futuros. Sin embargo, la percepción de los cambios en el clima está mediada culturalmente, como se muestra en el estudio comparativo de tres cadenas de montañas glaciares en los Andes del Perú, los Alpes italianos y del oeste de los EE.UU. (Brugger et al. 2013).

En la zona del presente estudio, el Instituto de Montaña (Vergara 2015) ha realizado entrevistas (89 en total) sobre las percepciones de los cambios en el clima que tienen residentes mayores de 40 años (59 hombres y 30 mujeres). Prácticamente todas las personas entrevistadas (94\%) afirman que el calor se ha incrementado, que la cantidad de agua disponible por las precipitaciones es menor (70\%) y que su intensidad también es menor (59\%); aunque un $20 \%$ considera que no hay cambio en los patrones de lluvia. Más de la mitad (55\%) percibe que se ha alterado la estacionalidad de las lluvias, siendo ahora más corto el periodo de lluvias y más largo el de sequía (Vergara 2015). El retroceso de glaciares figura de manera prominente como uno de los cambios mayores.

Pese a estas afirmaciones, no se identificaron cambios tecnológicos autónomos como respuesta al cambio percibido (Vergara 2015). Los cambios y adaptación en la zona de estudio se despliegan de una manera más holística, estableciéndose metas y desarrollando estrategias para construir su seguridad existencial, como muestra el estudio de Delgado (2006). Frente al cambio climático, la etnografía puede ayudar a entender la brecha que existe entre el saber tecnológico, la emoción existencial y las respuestas holística que construyen las familias pastoras, y el discurso tecnológico de la adaptación dirigida. Entender esta 
en que se están desarrollando respuestas desde la sociedad rural frente al cambio climático (Hassanein y Kloppenburg 2015).

Con la finalidad de ilustrar la importancia de la contextualización en el diseño de acciones de apoyo a los procesos de adaptación revisaremos el caso de dos comunidades alto-andinas ubicadas al sur de la Cordillera Blanca (Ancash, Perú). Estas comunidades están dedicadas a la ganadería en una de las zonas del mundo donde los efectos del cambio en los patrones históricos del clima han ocasionado, entre otras cosas, el retroceso acelerado de los glaciares que regulan la disponibilidad de agua, principalmente en la época de estiaje.

La contextualización se refiere a conocer mejor el entorno de trabajo, identificando características sociales, económicas y ambientales propias del lugar; y en este caso hemos usado la etnografía como una herramienta para contextualizar y poder encontrar pistas que cierren la brecha emocional, existencial y de significados entre la forma en que la población local responde a los cambios y la manera en que vemos estos cambios desde un enfoque externo y tecnológico.

En nuestra experiencia de trabajo en la Cordillera Blanca, las zonas altoandinas de puna —ubicadas a más de 3.800 m.s.n.m.-, son territorios que se están despoblando. Abandonar el lugar de origen es una respuesta dramática, que es parte de las estrategias de seguridad existencial a las que se refiere Delgado (2006). Estas respuestas tienen causas complejas, no solo relacionadas con las percepciones de cambios en el clima. Por ello, en este artículo se busca mostrar cómo diferentes aspectos de la vida del campesino y de su percepción están favoreciendo la migración y el despoblamiento de las partes más altas de los Andes. Para ello nos basamos en los resultados del trabajo etnográfico realizado entre enero y julio de 2017 en las comunidades campesinas Canray Grande y Los Andes de Recuay, ubicadas en la Cordillera Blanca, región Ancash, Perú.

\section{Problema de estudio y objetivos}

Durante nuestro acercamiento a los sitios de trabajo se identificó que las comunidades campesinas, y en particular la puna, se están despoblando. Existe una amplia literatura sobre la migración del campo a la ciudad (Matos Mar 1988 [1984]; Golte y Adams 1987), migración del campo al campo o nuevos focos económicos (Urrutia 1996a y 1996b, 1997, 1998; Glave y Pinedo 1997, Lozano 2006), y como parte del enfoque de nueva ruralidad, la migración de retorno (De Grammont y Martínez 2009; Diez 2014). Sin embargo, estas referencias se concentran principalmente en áreas agrícolas, ubicadas a menor altitud, y no toman en cuenta los flujos dentro de una misma comunidad, ni lugares de puna (ubicados a más de 3.800 m.s.n.m.). 
Aunque en las comunidades que trabajamos también se ha encontrado migraciones campo-ciudad, campo-campo y ciudad-campo, en la puna solo hemos visto migración sin retorno y un fuerte despoblamiento desde hace treinta años. Prueba de ello son los numerosos corrales y chozas abandonadas que hemos observado en la puna. En muchos casos, los pastores han fallecido sin que ningún familiar cercano los reemplace y utilice las chozas y corrales; y en otros, las familias se han mudado a las partes más bajas de la comunidad.

Ante esta situación de despoblamiento y flujos de migración nace la pregunta que buscamos responder: ¿por qué se está despoblando la puna?

\section{Método}

Esta investigación tiene una aproximación cualitativa que usa el método etnográfico, característico de la antropología. La etnografía busca entender el mundo desde el punto de vista de los actores o sujetos. Consiste en escuchar la voz del otro, en este caso los campesinos pastores de la Cordillera Blanca en Ancash. La etnografía busca describir e interpretar, sin caer en un paradigma establecido y favoreciendo nuevos descubrimientos (Guber 2011). Hay que tomar en cuenta, asimismo, que la descripción realizada por el investigador no es desde el mundo ni la percepción de los nativos, sino una interpretación que hace el investigador sobre lo que vio y escuchó (Guber 2011).

La etnografía, como método, se basa en el trabajo de campo. Aquí, el etnógrafo participa en la vida diaria de las personas durante un período de tiempo observando qué sucede, escuchando qué se dice, haciendo preguntas. El etnógrafo acopia todo dato disponible sobre el tema en que se centra la investigación (Hammersley y Atkinson 1994).

Por un lado, el investigador debe partir de una ignorancia metodológica y hacer el esfuerzo de aprender la realidad en términos no propios, planteando su interrogación a partir de preguntas abiertas, es decir aquellas que se limitan a pedir «explíqueme» (la expresión o idea que se ha escuchado); luego, describir e interpretar ('traducir') la cultura estudiada para un público que no necesariamente pertenece a dicha cultura. Por otro lado, el trabajo de campo implica una exposición del investigador a la realidad que estudia, por medio de las técnicas (entrevistas, observación participante) y el instrumento (el mismo investigador con sus atributos socioculturales) (Guber 2011). En la etnografía es muy importante ser un investigador reflexivo, que busque minimizar el efecto que genera en la cultura que estudia y describir los fenómenos tal y como son y no como meramente lo percibimos o como nos gustaría que fuera (Hammerley y Atkinson 1994). 


\subsection{La etnografía en nuestro trabajo}

Para esta investigación, se seleccionaron dos comunidades campesinas altoandinas ubicadas en la Cordillera Blanca, en las que el Instituto de Montaña ha estado trabajando y de las que se tenía información previa. La comunidad Canray Grande, que actualmente agrupa 320 familias, cuenta con 3.835 has de extensión en un rango de 3.500 a 4.450 m.s.n.m.; y la comunidad Los Andes de Recuay, que agrupa 64 familias, cuenta con 3.734 has de extensión entre 3.350 y 4.400 m.s.n.m. Ambas comunidades fueron creadas en la época de la Reforma Agraria, en territorios que antes estaban ocupados por haciendas, y conformadas por los antiguos arrendatarios. En ambos casos encontramos que menos del $5 \%$ de los comuneros tienen 35 años o menos y casi el $20 \%$ de los comuneros con menos de 70 años de edad se dedican a actividades no agropecuarias, fuera de su comunidad.

En ambos lugares, la mayor parte de las tierras comunales se han destinado a la producción ganadera de vacunos y ovinos; en Canray Grande representa el 77,2\% de la comunidad y en Los Andes Recuay el 93,5\%. La mayoría de las áreas ganaderas se encuentran en las partes más altas de cada comunidad, y están cubiertas por pastizales naturales y algunos humedales. La principal diferencia entre estas dos comunidades es que Canray Grande tiene un área destinada a la crianza de ganado que pertenece a toda la comunidad, además de áreas de aprovechamiento familiar, mientras que en Los Andes de Recuay, solo hay áreas de aprovechamiento familiar.

Siendo la ganadería la única actividad que se desarrolla en las partes altas (a más de 3.800 m.s.n.m.) de las comunidades elegidas, para el trabajo etnográfico se seleccionaron cuatro familias ganaderas de cada comunidad. Estas familias fueron elegidas para representar diferentes condiciones de la ganadería que se desarrolla en las comunidades, y se les hizo un acompañamiento durante días y noches para conocer de cerca la forma en que realizan el pastoreo de ovinos y vacunos, las características y forma de pensar de las familias, su forma de organizarse, entre otros. Es decir, tratar de conocer, con cierta profundidad, el mundo de los pastores.

Además del acompañamiento a las familias, se pasó tiempo en los diferentes caseríos que limitan con las comunidades de trabajo y en los que viven los comuneros. El trabajo de campo en cada comunidad duró en total un mes, durante los meses de lluvia (febrero y marzo); luego de procesar parte de la información, se visitó nuevamente a las familias elegidas durante la temporada seca (junio y julio).

Durante todo el trabajo de campo hubo que ser constantemente reflexivos para poder llevar de mejor manera la investigación, involucrando 
nuestra presencia y siendo conscientes de nuestra influencia en sus vidas cotidianas. Un aspecto importante a tomar en cuenta fue la calificación como 'ingenieros' que nos dieron los pastores. Con esta calificación los pobladores expresan su respeto, pero también marcan distancia entre el nosotros pastores, comuneros, locales, con pocas posibilidades y muchas veces sin educación, y los otros ingenieros y profesionales que son foráneos, extraños, de la ciudad, etcétera.

Esta reflexividad nos permitió redireccionar las reacciones de los actores y utilizarlas mejor. Por ejemplo, en muchas entrevistas grabadas los pastores comentan solo una parte de lo que piensan o expresan algunas ideas que se alejan de su realidad, porque sienten desconfianza hacia el extraño que le cuestiona sobre aspectos de su familia, sus actividades y su vida en general. Sin embargo, la convivencia y pasar tiempo con las familias permite ganar mayor confianza mutua y crear momentos de conversación informal donde temas o preguntas que antes produjeron desconfianza ya no lo hacen, y ahora el informante cuenta su vida con veracidad.

En este sentido, el trabajo de campo etnográfico nos ha permitido también validar la información obtenida. La convivencia y las conversaciones informales permitieron validar constantemente lo que dicen (el discurso) y lo que hacen (la praxis) las familias ganaderas en nuestra área de estudio. También se accedió a información general sobre la comunidad; se pudo conversar largamente con ancianos en cada zona, que compartieron sus memorias y experiencias sobre cómo era la puna hace 30 años o más y los cambios que han percibido.

En general, las técnicas de recojo de información utilizadas fueron la entrevista semiestructurada y la observación participante; ambas técnicas realizadas en la convivencia con los pastores y en los caseríos visitados, dentro del trabajo de campo etnográfico.

\section{Resultados}

Todas las familias estudiadas manejan un gradiente altitudinal, con al menos dos zonas de producción: en la parte baja un área principalmente agrícola y en la puna o parte alta un área ganadera. Durante la temporada de lluvias (de diciembre a marzo) viven en la puna, para pastorear y cuidar sus animales; y durante la temporada seca (de abril a noviembre) viven en la parte baja, para poder cumplir con las actividades agrícolas de siembra, aporque (setiembre a noviembre) y cosecha (mayo a julio). Además, los rastrojos y restos dejados después de la cosecha y las tierras agrícolas en descanso son útiles para 
alimentar al ganado en esta época de estiaje. Con esta rotación se permite, además, el descanso y recuperación de los pastos en la puna.

Las observaciones realizadas durante el trabajo de campo etnográfico nos han permitido identificar dos factores que no se relacionan con los cambios en las condiciones climáticas y que están influyendo en el abandono y despoblamiento de la puna: el ciclo demográfico de la familia pastora y las percepciones sobre el pastoreo de pobladores de diferentes generaciones.

\subsection{Ciclo demográfico de las familias pastoras y estrategias de vida}

Identificamos tres tipos de familia, relacionados con los tres períodos por los que puede pasar una familia pastora. El primero es el periodo de formación, cuando la pareja se casa y todavía no tiene hijos, o tiene hijos pequeños; la edad de la pareja oscila entre 18 y 30 años. Luego viene el período de consolidación de la familia; aquí la edad de la pareja oscila entre los 30 y 60 años, los hijos ya han crecido - la mayoría tiene más de 12 años-y algunos se encuentran estudiando en la ciudad, y los hijos mayores -incluso- están formando su propia familia. Por último, está el período del decrecimiento de la familia. Aquí, la pareja ha envejecido, tiene 60 años o más, y a veces alguno de los cónyuges ya ha fallecido; la mayoría de los hijos están fuera del hogar de sus padres, a veces viviendo en la ciudad y están consolidando su propia familia. En cada etapa, la familia utiliza estrategias de vida, siguiendo objetivos distintos y correspondientes al ciclo en el que se encuentra. Aquí utilizamos el concepto de estrategias de Annalies Zoommers.

Las estrategias de vida refieren a la manera en que una familia responde a cambios en la relocalización de recursos de tierra, trabajo y capital. Ante estos cambios, la familia responde combinando sus oportunidades y limitaciones, en base a sus prioridades u objetivos. Estas oportunidades y limitaciones son creadas por el contexto externo (situación agroecológica, acceso al mercado, infraestructura y grado de organización) y a la disponibilidad de recursos a nivel familiar (fuerza de trabajo, tierra, agua, ganado y capital) (Zoomers 1998: 15; Zoomers 2002: 30).

Es decir, una familia, sobre la base de sus objetivos, moviliza y combina sus oportunidades y limitaciones en cada contexto en particular. De acuerdo con el momento en que se encuentra desarrolla estrategias de acumulación, consolidación o superación de vulnerabilidades. 


\subsection{Momentos de la familia que usa la puna}

\subsubsection{Familia en formación: El caso de Nérida [28] y Julio [30] - Canray Grande}

Nérida tiene 28 años y Julio 30, ambos tienen cinco hijos, que tienen entre 1 y 11 años. Durante la temporada de lluvias la familia vive en la puna. Julio vive con su esposa pero todos los días baja a la sede de la comunidad o a los pueblos cercanos en busca de algún trabajo temporal (cachuelo). Mientras tanto, Nérida se queda constantemente en la puna para atender a sus hijos, pastorear los animales y dedicarse a los quehaceres del hogar; en raras ocasiones baja al pueblo o a la ciudad, para hacer algunas compras. Los hijos mayores (de 10 y 11 años), ayudan en las actividades de pastoreo y a cuidar a sus hermanos menores.

Nérida pastorea en total 184 ovinos y 24 vacunos, de los cuales son propios solo 25 ovinos y 11 vacunos, el resto pertenece a otras seis personas, miembros de su familia o de la comunidad. Con los dueños de los animales se tienen diferentes arreglos: la modalidad de encargo, donde el dueño da algún pago o permiso para que se usen sus pastos a cambio del cuidado de los animales, y la de arriendo, donde el dueño de los animales y el que los cuida se dividen en partes iguales las crías que tiene el ganado en una temporada. A esto se le llama 'crías al partir'.

\section{Cuadro 1}

\begin{tabular}{|l|c|c|l|}
\hline \multicolumn{1}{|c|}{ Dueño } & Ovinos & Vacunos & \multicolumn{1}{c|}{ Arreglo } \\
\hline Propio & $\mathbf{2 5}$ & $\mathbf{1 1}$ & \\
\hline Mamá & 18 & 4 & Encargo (uso de pastos) \\
\hline Hermana & 31 & 1 & Crías al partir \\
\hline Tía Anatolia & 25 & - & Crías al partir \\
\hline Señor de Paz & 18 & - & Encargo (uso de pastos + pago en dinero) \\
\hline Señor Ponte & 34 & 8 & Encargo (uso de pastos + pago en dinero) \\
\hline Señor Coral & 33 & - & Encargo (uso de pastos + pago en dinero) \\
\hline
\end{tabular}

Elaboración propia a partir de entrevista y acompañamiento a una familia joven. 


\section{Estrategias de acumulación}

Como hemos visto, Nérida y Julio tienen poco ganado propio, ya que llevan pocos años en la actividad de pastoreo. Esta familia, aprovechando su juventud desarrolla una serie de estrategias que les permiten alcanzar el objetivo de acumular ganado propio:

- $\quad$ Generalmente se inicia con pocos animales que son heredados de sus familiares.

- $\quad$ Pastan animales de otros por encargo, lo que les permite usar el pasto de otros para sus propios animales.

- Pastan animales de otros por arrendamiento, lo que les permite aumentar el tamaño del rebaño propio al quedarse con las crías.

En otras familias se ha visto, además, que se emplea la migración temporal como una estrategia para acumular capital que después se invierte en la adquisición de bienes, como chacras, terrenos, construcción de vivienda, entre otros.

\subsubsection{Familia consolidada: El caso de Teodora [54] y Clemente [50] - Canray Grande}

Teodora tiene 54 años y Clemente, su esposo, 50. Tienen seis hijos que tienen entre 15 y 30 años. Los tres hijos mayores ya han formado su propia familia y dos de ellos viven en ciudades fuera de la comunidad (en Huaraz y Lima). Los otros cuatro hijos viven junto a sus padres, incluyendo la hija mayor con su esposo y sus dos hijas pequeñas, aunque esta familia joven está en proceso de independizarse.

Clemente reside principalmente en la casa que tienen en Huaripampa (poblado aledaño al territorio de la comunidad), ocupa su tiempo como dirigente de la comunidad, en las actividades agrícolas y, cuando puede, algún trabajo temporal. Algunos días sube a la puna para acompañar a su esposa y ayuda arreglando los corrales o la choza en la que duermen. Mientras tanto, Teodora vive permanentemente en la puna y se encarga de los animales.

Los hijos viven en Huaripampa, junto a Clemente. La hija mayor tiene un empleo y cuida su propia familia, además de apoyar a sus padres en la cocina, en la chacra y en otras actividades. Los otros tres hijos estudian en Huaripampa pero los fines de semana y durante las vacaciones escolares suben a la puna a ayudar a su madre en el pastoreo; cuando terminen la secundaria se irán a estudiar a Huaraz. 
Teodora pastorea un total de 224 ovinos y 12 vacunos. De estos, 122 ovinos y 8 vacunos son propios, y el resto son encargados y pertenecen a otros ocho dueños. Como tienen muchos ovinos propios, cada cierto tiempo invierten en la compra de padrillos (machos, sementales de raza mejorada) para mejorar la calidad del ganado ovino. Además, mediante los arreglos hechos han conseguido acceder a tres sectores diferentes de pastos en la puna.

\section{Cuadro 2}

\begin{tabular}{|l|c|c|l|}
\hline \multicolumn{1}{|c|}{ Dueño } & Ovinos & Vacunos & \multicolumn{1}{c|}{ Arreglo } \\
\hline Propio & $\mathbf{1 2 2}$ & $\mathbf{8}$ & \\
\hline Mamá & 20 & 2 & Ninguno \\
\hline Suegra & 10 & - & Ninguno \\
\hline Vecino & 8 & 2 & Encargo (pago en dinero) \\
\hline Señor Ponte & 24 & - & Encargo (pago en dinero) \\
\hline Señora Sarmiento & 9 & - & Encargo (pago en dinero) \\
\hline Primo & 6 & - & Encargo (uso de pastos) \\
\hline Hermana & 20 & - & Encargo (uso de pastos) \\
\hline Señora Poma & 5 & - & Encargo (uso de pastos) \\
\hline
\end{tabular}

Elaboración propia a partir de entrevista y acompañamiento a una familia consolidada.

\section{Estrategias de consolidación}

Teodora y Clemente ya han acumulado ganado propio y no tienen animales arrendados, pero siguen aprovechando de los beneficios del uso de otros pastos que genera el tener animales encargados. Su objetivo es consolidarse y mejorar sus activos y para ello desarrollan estrategias para invertir parte de sus ganancias en:

- El mejoramiento del ganado con la introducción de padrillos mejorados.

- La ampliación de áreas de pastoreo para realizar la rotación.

- Siembra de pastos cultivados para mejorar la alimentación del ganado.

- $\quad$ Inversión en educación superior (instituto o universidad) de sus hijos en Huaraz.

Además, en otras familias se ha visto que invierten en la compra de más chacras y terrenos. Algunos compran un terreno o casa en la ciudad donde viven sus hijos y les permite a ellos mismos desarrollar alguna actividad en la zona urbana. 


\subsubsection{Familia anciana: el caso de Isidora [68] - Los Andes}

Isidora ha abandonado la puna. Tiene 68 años y quedó viuda hace varios años. Tiene diez hijos que tienen entre 27 y 55 años. Ocho de ellos ya tienen su propia familia, y la mayoría vive fuera de la comunidad (Argentina, Huaraz, Canrey Chico, y Recuay). Solo dos viven junto a su madre, uno es soltero y se emplea en cualquier trabajo eventual que encuentra, y la otra tiene su propia familia (cinco hijos y dos nietos), y se dedica a atender la casa mientras el esposo trabaja en la selva y la visita cada seis meses.

Isidora se encarga de pastorear todos los días el ganado, pero sus nietos la ayudan durante los fines de semana y vacaciones escolares. Ellos también ayudan con las labores de la casa, ya que Isidora se está recuperando de una enfermedad.

Antes Isidora vivía en la puna y tenía más de cien ovinos propios, pero a medida que fue envejeciendo, fue reduciendo el número de animales y dejó la puna. Ahora vive en Ocopampa, un poblado que limita con la parte baja de la comunidad Los Andes al que se mudó porque -inicialmente- su esposo conseguía con frecuencia trabajos bien remunerados en las ciudades cercanas (Recuay, Cátac, Ticapampa) y se quedó ahí una vez que su esposo falleció.

Ahora Isidora tiene pocos animales propios (15 ovinos y 1 vaca), pero pastorea en total 120 ovinos y 2 vacunos de diez dueños diferentes, la mayoría de los cuales son sus familiares. Ella piensa seguir así unos años más y luego dejar la actividad a su hija, si es que ella quiere. Si no, devolverá todos los animales a sus dueños y los animales propios los encargará a algún pastor en la puna.

\section{Cuadro 3}

\begin{tabular}{|l|c|c|l|}
\hline \multicolumn{1}{|c|}{ Dueño } & Ovinos & Vacunos & \multicolumn{1}{c|}{ Arreglo } \\
\hline Propio & $\mathbf{1 5}$ & $\mathbf{1}$ & \\
\hline Hija Estelista y suegra & 20 & - & Ninguno \\
\hline Hijo Severo & 7 & - & Ninguno \\
\hline Hijo Santiago & 3 & - & Encargo (pago en dinero) \\
\hline Hijo Tito y suegra & 27 & - & Crías al partir \\
\hline Hijo Eugenio y suegra & 10 & 1 & Encargo (pago en dinero) \\
\hline Hermano Grimaldo & 15 & - & Encargo (pago en dinero) \\
\hline Vecina Sofía Trejo & 9 & - & Encargo (pago en dinero) \\
\hline Sobrina Macaria & 7 & - & Encargo (pago en dinero) \\
\hline Sobrina Manuela & 7 & & Encargo (pago en dinero) \\
\hline Sobrino Yefer & 3 & - & Encargo (pago en dinero) \\
\hline
\end{tabular}

Elaboración propia a partir de entrevista a y acompañamiento a una familia anciana. 


\section{Estrategias de compensación y superación de vulnerabilidades}

Isidora, debido a su edad y circunstancias adversas como la muerte de su esposo y su propia enfermedad, ha desarrollado una serie de estrategias con el objetivo de conseguir ingresos para superar las vulnerabilidades:

- $\quad$ Reducción de su ganado, vendiendo una gran parte del rebaño propio.

- $\quad$ Migración definitiva a la parte baja de la comunidad.

- Reciprocidad, recibiendo apoyo familiar, especialmente de los que encargan su ganado.

- Planifica vender casi todo su ganado, lo que resta lo encargaría a otro pastor.

Hemos encontrado, además, que otras familias ancianas también venden o alquilan sus bienes (chacras) porque ya no pueden aprovecharlas y necesitan ingresos.

\section{Estrategias de reducción de riesgos}

Las familias ganaderas, además de las estrategias descritas que se relacionan con los objetivos específicos que se tienen en cada etapa del ciclo familiar, desarrollan estrategias de reducción de riesgos. La mayoría busca diversificar sus actividades e ingresos para reducir el riesgo de pérdida económica, aprovechando el contexto agroecológico al que tienen acceso y la cercanía a centros urbanos (fuentes de trabajo). En algunos casos, la diversificación se observa dentro de la actividad ganadera, diversificando los animales criados, invirtiendo más en los cuidados y calidad del ganado, para obtener diferentes productos derivados (lana, carne, leche, queso, etcétera). También hay estrategias de ahorro en dinero o en bienes, guardados para situaciones de crisis.

\subsection{Ciclo de vida orientado al despoblamiento}

A través de los estudios de caso se ha mostrado el ciclo de las familias ganaderas en las comunidades en que trabajamos. En estas comunidades, el promedio de edad de todas las personas inscritas como comuneros, no solo los ganaderos, supera los 60 años, y en general son pocas las familias jóvenes que se quedan en la comunidad. Hemos encontrado también que en las comunidades estudiadas cada vez son más las familias que se encuentran en la etapa de envejecimiento y menos en la etapa de formación. 
El ciclo demográfico presentado muestra a las familias desarrollando distintas estrategias en cada etapa del ciclo. Todas las familias crían ganado propio y de otras personas, sin embargo, la proporción varía de acuerdo con el ciclo de vida. En un principio acumulan o consiguen ganado, y crían mayor cantidad de ganado de otros bajo formas de arrendamiento y encargo. Luego se consolidan y logran tener mayor ganado propio. Sin embargo, conforme la familia envejece, disminuye el número de ganado propio y abandonan la puna. Este abandono se da por distintas razones: prefieren relacionarse más con la ciudad por nuevas ocupaciones, educación de sus hijos, o quedan viudos y ya no tienen la misma fuerza para vivir en la puna. Al mismo tiempo, sus hijos buscan relacionarse más con la ciudad; ningún hijo de la familia consolidada o en decrecimiento vive en la puna. En general, el ciclo de vida actual de las familias pastoras conduce al abandono de la puna.

\section{Gráfico 1}

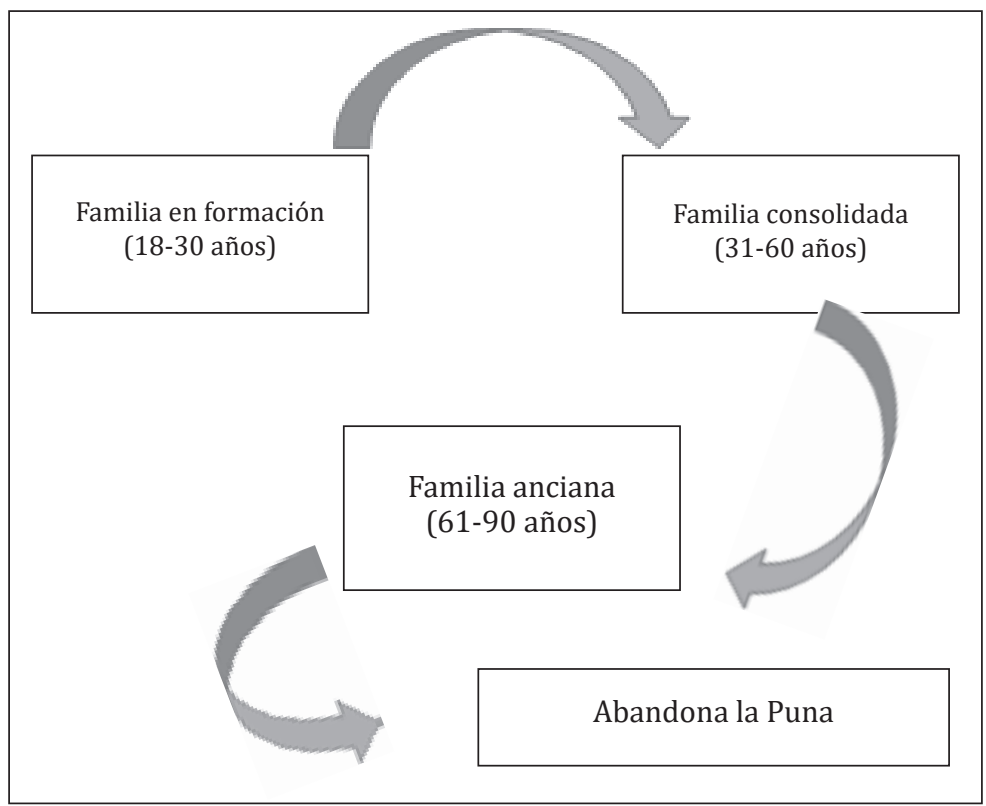

Fuente: Trabajo de campo.

Elaboración propia.

Entonces, ya no se da el ciclo de vida que permite la residencia y permanencia de las familias en la puna como muestra un estudio sobre pastores en el distrito de San Marcos, callejón de Conchucos (Salas 2008). Dicho estudio muestra que la puna es expulsora de mano de obra; es decir, los hijos deben salir porque no alcanzan los recursos (pastos) para todos; pero no todos salen, sino que tienen un ciclo de vida donde alguno de los hijos 
se queda a vivir en la puna remplazando a sus padres, quienes se quedan a vivir hasta sus últimos días en la puna (Salas 2008). En nuestro caso, por el contrario, existe un ciclo de vida donde los ancianos abandonan la puna y sus hijos ya no los remplazan.

\subsection{Percepciones sobre el pastoreo}

Reconocer las percepciones que tienen las personas de la comunidad (no solo los ganaderos) sobre la actividad de pastoreo, nos ayuda a entender por qué cada vez menos familias jóvenes continúan con la ganadería en la puna. Además nos muestra las fuertes diferencias entre generaciones sobre la valoración del trabajo en la puna. La vida del pastor en la puna es vista, en general, como una vida muy sacrificada por las difíciles condiciones climáticas que hay que tolerar; mucho frío en las noches, fuertes vientos y heladas en la época seca, y peligrosas tormentas eléctricas en la época de lluvia. Además, el pastoreo diario de los animales requiere caminar, en algunas ocasiones varios kilómetros para encontrar buenos pastos. Todo esto hace que el pastoreo sea visto como una actividad temporal, de corto o mediano plazo y en ningún caso observado como una actividad transgeneracional.

Los ancianos en las comunidades perciben el pastoreo en la puna como una actividad sacrificada que requiere la salud y juventud que a ellos les falta. Como ya no soportan ese ritmo de vida, optan por irse a lugares más bajos y criar animales en menor número. Algunos prefieren encargar algunas cabezas de ganado a quienes aún se dedican al pastoreo, y otros abandonan completamente la ganadería.

Ya no ya. Ya no hay salud, ya no permite frío, lluvia, tanto que he sufrido (Anciano de 77 años que antes tenía ganado pero ahora ha abandonado la puna).

Ellos [sus hijos en la ciudad] no quieren que estemos acá. Reniegan. Porqué están en el frío, en la lluvia. Sufren mamá, sufren papá. Siempre me llaman y me gritan. Ya, ya, ya les digo. [...] Unos años más y me voy abajo, mucho sacrificio, ya estamos cansados (Pastor de 65 años).

Los adultos consolidados perciben la ganadería como una actividad importante, que les genera ingresos para comprar víveres, ropa, pagar los estudios superiores de sus hijos, entre otros. Sin embargo, tampoco consideran el pastoreo como una actividad a largo plazo. La mayoría piensa conservar sus animales y quedarse en la puna solo hasta que sus hijos terminen los estudios 
superiores. Hay quienes tienen planeado mudarse a un poblado cercano a la comunidad para tener una vida más tranquila y menos sacrificada que en la puna; criar ahí ganado mejorado en pequeña cantidad, sobre todo vacunos, y sembrar pastos cultivados (avena, raigrás, etcétera.). Otros piensan abandonar la ganadería, o tal vez encargar algunas cabezas de ganado, y cambiar de actividad, por ejemplo el comercio (poner una bodega), y estar al lado de sus hijos. También tienen planeado irse a vivir a ciudades grandes como Huaraz o Lima.

[...] los ovinos necesitan más salud para cuidar [...]. Yo voy a sembrar pasto mejorado y más dedicar en lo que es lechería [...]. Si lo terminamos de educar a nuestros hijos [en la universidad y el colegio] lo bajamos [se mudan a vivir a la parte baja], pocos ovinos en un potrero y vacas lecheras vamos comprar de buenas razas y con eso vamos a producir como pa' vender. Nuestros vacunos lo vamos meter en pastos mejorados y ya no vamos caminar como ahora (Adulto de 50 años que pastorea en la puna).

[...] criar acá hasta poder sacar nuestros hijos profesionales. Ya de ahí ya, tenemos que bajar ya a Canray (Pastora de 38 años).

En comparación, los jóvenes consideran al pastoreo como una actividad muy sacrificada, que los mantiene en la pobreza. Ninguno de los entrevistados tiene como prioridad quedarse a pastorear en la puna. Dentro de sus objetivos más importantes está el conseguir un empleo en la ciudad, y para ello es importante estudiar y tener una profesión; aunque algunos consideran que pueden encontrar otro tipo de trabajo o dedicarse al comercio. Conseguir un trabajo en la ciudad les permitiría salir y estar lejos de la puna, y alcanzar las comodidades que brinda la ciudad.

En el caso que fracasen en la ciudad, algunos no descartan regresar a la comunidad, pero a los poblados cercanos a la comunidad, y eventualmente encargar sus animales a alguien más en la puna.

Terminando el colegio estudiaré pues. Trabajar y después estudiar, cómo será [...] en Huaraz, para secretaria. Estoy pensando eso [...]. No me gusta [vivir en la puna] jajaja. Es que hace frío pue, mucha lluvia. A mi mamá sí le puede gustar. Nos vamos, después venderán pues, hasta que acabe de estudiar el colegio no más pues. (Estudiante de $5^{\circ}$ año de secundaria, ayuda a pastorear en la puna durante vacaciones escolares).

Pienso quedarme unos cinco añitos más. Después de eso hacer algo bueno ya por la vida [...]. Siquiera unos 200, 300 ovejas o algo más 
[acumular]. Venderlo todito, tener capital y poner una farmacia o una ferretería en Huaraz, Carhuaz, o algo por el callejón. (Joven de 26 años que pastorea en la puna).

Así, cada vez menos familias habitan la puna y muchas de las que lo hacen ven en la ganadería una opción para acumular suficientes recursos para irse lejos, y asegurar que ellos o al menos sus hijos tegan una vida mejor. Todas las condiciones son agravadas por los efectos del cambio climático. Hemos visto que en las partes altas de estas comunidades no solo se ha perdido masa de los glaciares, sino que este retroceso ha permitido un proceso de contaminación natural (por intemperismo) de sus fuentes de agua. Asimismo, se ha incrementado la frecuencia de las tormentas eléctricas en la puna, registrándose casi en cada temporada de lluvia la muerte de algún comunero o miembro de su familia provocada por la caída de rayos. Todo esto sin contar con el incremento de la época de sequía, que afecta principalmente la cantidad y calidad de pastos naturales disponibles para alimentar a su ganado, obligándolos a caminar mayores distancia en la búsqueda de pastos para sus animales.

\section{Conclusiones}

Encontramos un proceso de despoblamiento de las zonas de puna o regiones altoandinas (a más de 3.800 m.s.n.m.). En las comunidades estudiadas, encontramos evidencias del abandono de áreas que antes eran usadas para el pastoreo del ganado. Para entender este proceso, hemos hecho un retrato de las familias que actualmente son ganaderas, describiendo el ciclo de vida de las familias, cuyo objetivo final es acumular lo suficiente para poder abandonar la puna y darle a sus hijos mejores condiciones de vida de las que han tenido los padres. Además, encontramos entre los jóvenes una sensación de rechazo hacia las condiciones de vida de la puna y a las actividades de pastoreo. Esto ha generado que las personas más ancianas de la comunidad abandonen la puna porque no tiene fuerzas para mantener ese estilo de vida y que no haya nuevos jóvenes que los reemplacen.

En este sentido, la etnografía es una herramienta potente para contextualizar el cambio climático. La etnografía nos permite reconstruir casos que ilustran-a través de la selección de textos y narrativa de los propios actores-el contexto complejo de dimensiones biofísica, social, económica, cultural y emocional en el que se incrustan como elementos de un todo más complejo los efectos directos e indirectos del cambio climático. 


\section{Referencias}

BRugger, Julie, K. W. Dunbar, Christine Jurt y Ben Orlove (2013). «Climates of anxiety: Comparing experience of glacier retreat across three mountain regions». Emotion, Space and Society, 6 (February), 4-13. https://doi.org/10.1016/j. emospa.2012.05.001

Delgado Herrera, A. (2006). «"I want to be somebody". Building and Experiencing Existential Security in Two Andean Commuities». Tesis de Maestría no publicada. Vrije Universitet Amsterdam, Department of Social and Cultural Anthropology.

De Grammont, H. y L. Martínez (2009) La pluriactividad en el campo latinoamericano. Quito: FLACSO.

DiEz, A. (2014). Cambios en la ruralidad y cambios en las estrategias de vida en el mundo rural. Una relectura de antiguas y nuevas definiciones. SEPIA XV. Lima: SEPIA, pp. 19-85.

GlaVe, M. y D. Pinedo (1997). De la Puna a la montaña: migración y uso de espacio en el valle de Inambari. Debate agrario. Lima, número 26, pp. 19-40.

Golte, J. y N. AdAms (1987). Los caballos de troya de los invasores: estrategias campesinas en la conquista de la gran Lima. Lima: IEP.

Guber, R. (2011). La etnografía, Método, Campo y Reflexividad. Buenos Aires: Siglo XV Editores

HAMmersley, M. y P. ATKInSON (1994). Etnografía: método de investigación. Barcelona: Paidós.

KLopPenbuRG J. R. Jr.y N. HaSSANEIn (2015). «Where the Grass Grows Again: Knowledge Exchange in the Sustainable Agriculture Movement». Rural Sociology, 60, December, pp. 721-740.

LozANo, M. (2006). «Elementos para la clasificación de estrategias familiares campesinas en el Alto Tambopata». Debate Agrario. Lima, número 40-41, pp. 85-98.

Matos MaR, J. (1988 [1984]). Desborde popular y crisis del estado: el nuevo rostro del Perú en la década de 1980. Lima: CONCYTEC.

SALAS, G. (2008). Dinámica social y minería: familias pastoras de puna y la presencia del proyecto Antamina. Lima: IEP.

Urrutia, J. (1996a). Relaciones laborales, empleo agrícola y sociedad rural en Cajamarca. Debate Agrario. Lima, número 24, pp. 93-114.

URRUTIA, J. (1996b). Relaciones laborales y sociedad rural: Huamanga y Huanta. Debate Agrario. Lima, número 25, pp. 1-19.

UrRutia, J. (1997). Relaciones laborales y sociedad rural en Cusco. Debate Agrario. Lima, número 26, pp. 1-18.

Urrutia, J. (1998). Relaciones laborales y sociedad rural en Chincha y Huaral. Debate Agrario. Lima, número 27, pp. 1-24. 
REVISTA KAWSAYPACHA: SOCIEDAD Y MEDIO AMBIENTE $N^{\circ} 2$ (2018)

VERGARA K. (2015). Línea de base y metodología para analizar riesgos, factores de vulnerabilidad y capacidades adaptativas locales. Informe no publicado. Lima: Instituto de Montaña.

Zoomers, A. (1998). Estrategias campesinas en el Sur Andino de Bolivia: intervenciones y desarrollo en el norte de Chuquisaca y potosí. La Paz: CEDLA.

Zoomers, A. (2002). Vinculando estrategias campesinas al desarrollo: experiencias en los andes bolivianos. La Paz: Plural editores. 